\title{
QUALITY IMPROVEMENT Shifting the focus: A QI project to improve the management of delirium in patients with hip fracture
}

\author{
Authors: Letitia Dormandy, ${ }^{\mathrm{A}}$ Sana Mufti, ${ }^{\mathrm{B}}$ Emma Higgins, ${ }^{\mathrm{C}}$ Cate Bailey ${ }^{\mathrm{D}}$ and Martha Dixon ${ }^{\mathrm{E}}$
}

\section{Introduction}

Delirium is common in the perioperative setting, particularly in those admitted with a neck of femur fracture. It is associated with poorer outcomes, including increasing mortality, morbidity and prolonged hospital stay. It is often poorly recognised and under diagnosed.

Setting

An urban district general hospital.

Intervention

A steering group was set up and used 'plan, do, study, act' methodology to develop a diagnostic pathway and educational programme for all staff working with patients admitted with neck of femur fracture.

Results

There was an increase in the multidisciplinary teams use of the 4 AT delirium screening tool by $26 \%(p=0.0008)$. Staff surveys indicated an increase in the knowledge of delirium and confidence at explaining it to patients.

Discussion

By increasing staff confidence and use of recognised screening tools it is hoped that accurate diagnosis of this perioperative complication is improved, leading to improved management of these complex patients.

KEYWORDS: Neck of femur fracture, delirium, perioperative medicine

\section{Introduction}

Delirium in the perioperative setting is a common condition, although incidence varies depending on the type and timing of the operation. ${ }^{1}$ Those admitted with a neck of femur (NOF) fracture have one of the highest incidences reported; with between $30-65 \%$ of patients affected. ${ }^{1}$ The presence of delirium postoperatively has significant prognostic and economic effects and is associated with higher mortality and greater functional

Authors: ${ }^{A}$ geriatric medicine registrar, Homerton University Hospital NHS Foundation Trust, London, UK; ${ }^{B}$ consultant geriatrician, Homerton University NHS Foundation Trust, London, UK; Clead nurse in dementia care, Homerton University Hospital NHS Foundation Trust, London, UK; ${ }^{D}$ psychiatry registrar, Homerton University Hospital NHS Foundation Trust, London, UK; ${ }^{\text {E }}$ clinical nurse specialist in orthopaedics, Homerton University Hospital NHS Foundation Trust, London, UK decline. ${ }^{2}$ In addition, patients with delirium have been shown to have higher per-day hospital costs, longer length of stay and higher rates of nursing home placement. ${ }^{3}$

Delirium cannot be diagnosed using specific clinical biomarkers or diagnostic tests. It is often poorly recognised and under diagnosed. ${ }^{4}$ A number of screening tools have been developed to help in the assessment including $4 \mathrm{AT}$ and single question in delirium (SQiD). ${ }^{5}$ The SQiD tool states that a friend or family member of the patient should be asked the question: 'Is the patient more confused or drowsy than normal?' If the answer is yes then it is suggestive of delirium. This was first validated as a screening tool in 2001. ${ }^{6}$ The 4 AT was first developed in Edinburgh in 2011 with the most recent version published in 2014. ${ }^{5}$ It has been validated in a number of settings including general medical wards, emergency departments, preoperatively and in multicentre trials. It was found to be a valid screening tool even with limited training and when used by different health professionals, and is part of the National Institute for Health and Care Excellence (NICE) guidelines for delirium management. ${ }^{7}$

Treatment of delirium involves managing the underlying cause and preventing exacerbating factors. A commonly used mnemonic to help medical professionals identify and treat these factors includes the PINCH ME mnemonic (Box 1$)^{8}$

\section{Aim}

Delirium is often poorly recognised leading to suboptimal management. This was identified as a concern at Homerton University Hospital, a district general hospital in east London. A quality improvement project was consequently developed aiming to improve healthcare professionals' (therapy, nursing, medical) knowledge and confidence in diagnosing and managing delirium on inpatient surgical wards.

\section{Box 1. PINCH ME mnemonic}

Pain

Infection

Nutrition

Constipation

Hypoxia/hydration

Medication

Environment 
Fig 1. Three-step pathway for the assessment and management of delirium.

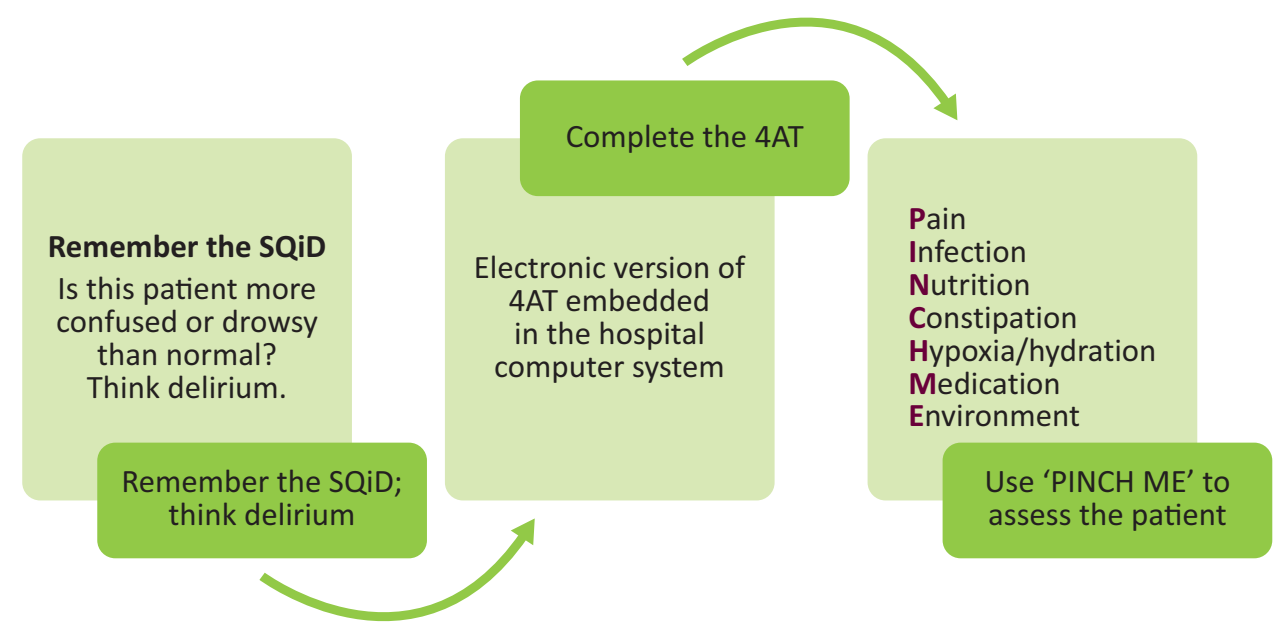

\section{Methods}

The project was completed at a 343-bedded district general hospital in the London borough of Hackney. The intervention was carried out on all surgical wards ( $n=2)$ accepting emergency surgical admissions. These wards included patients under the care of a wide range of surgical specialties; however, the project focused on those admitted with NOF fracture.

The project followed 'plan, do, study, act' (PDSA) methodology. ${ }^{9}$ A steering group, led by a consultant geriatrician, was set up to complete the project. Other members included a psychiatry registrar, a geriatric medicine registrar, specialist nurses (in dementia and orthopaedics), a physiotherapist, an occupational therapy assistant and surgical ward managers. The steering group developed a three-step pathway for the assessment and management of delirium (see Fig 1) over a 2-month period. This was based on national guidelines which were adapted to meet local needs. A strategy for its delivery was devised, including embedding 4AT into the patients' electronic records (Cerner) and an educational programme for clinical staff (see Fig 2). This was implemented in December 2017.

The effectiveness of the intervention was assessed using a review of the electronic notes system (Cerner). All patients admitted to the surgical wards (at any point during their admission) with a NOF fracture, in the 6 months (January-June 2018) post implementation were included in the analysis. This was compared to a control group of patients. Patients were included in the control group if they had been admitted to the surgical wards (at any point during their admission) with a NOF fracture in the 6 months of January-June 2017. This control group was selected in order to minimise the variation that is likely to occur in this group of patients and the number of admissions in summer and winter months.

The primary (process) measure was the use of the 4AT. Secondary measures included outcome measures comprising key performance indicators; length of stay, mortality, change in discharge destination (not including increase or change in care package) and coding on the discharge summary.

Staff confidence was also measured pre- and post intervention via questionnaires. Control group data were collected throughout 2017. Post-intervention group data were collected after small group lectures in December 2018. Staff were tested on their ability to name delirium risk factors (as defined by inclusion in the NICE guidelines and or PINCH ME mnemonic) and confidence regarding delirium (diagnosis and explanation) using a Likert scale (1-5). In addition, doctors were asked to name any local or national guidelines.

Data were analysed using GraphPad Prism software.

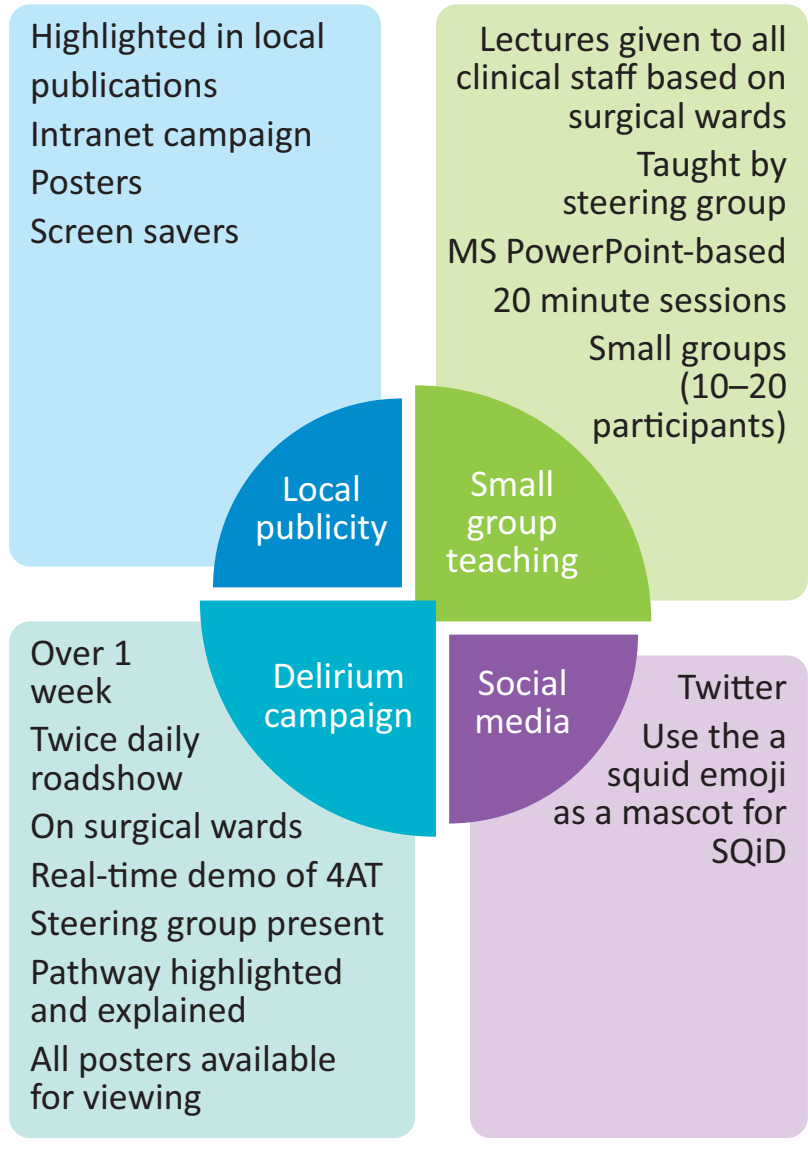

Fig 2. Implementation strategy: launched in December 2017. 
Table 1. Comparative demographic data for control and intervention groups

\begin{tabular}{|c|c|c|c|c|}
\hline & $\begin{array}{l}\text { Control group } \\
\text { (January-June 2017) } \\
n=40\end{array}$ & $\begin{array}{l}\text { Intervention group } \\
\text { (January-June 2018) } \\
n=45\end{array}$ & $\mathrm{p}$ value & Statistical test \\
\hline Age, mean (SD) & $79.1(60-98)$ & 82.68 (60-99) & 0.1258 & t-test \\
\hline Female, \% & 65 & 73 & 0.4053 & $\chi^{2}$ \\
\hline Number of comorbidities, mean & 3.68 & 3.77 & 0.8554 & Mann-Whitney U \\
\hline Delirium risk factors, ${ }^{a}$ mean & 2.6 & 2.78 & 0.4699 & Mann-Whitney $U$ \\
\hline Pre-existing cognitive impairment, \% & 15 & 20 & 0.5461 & $\chi^{2}$ \\
\hline
\end{tabular}

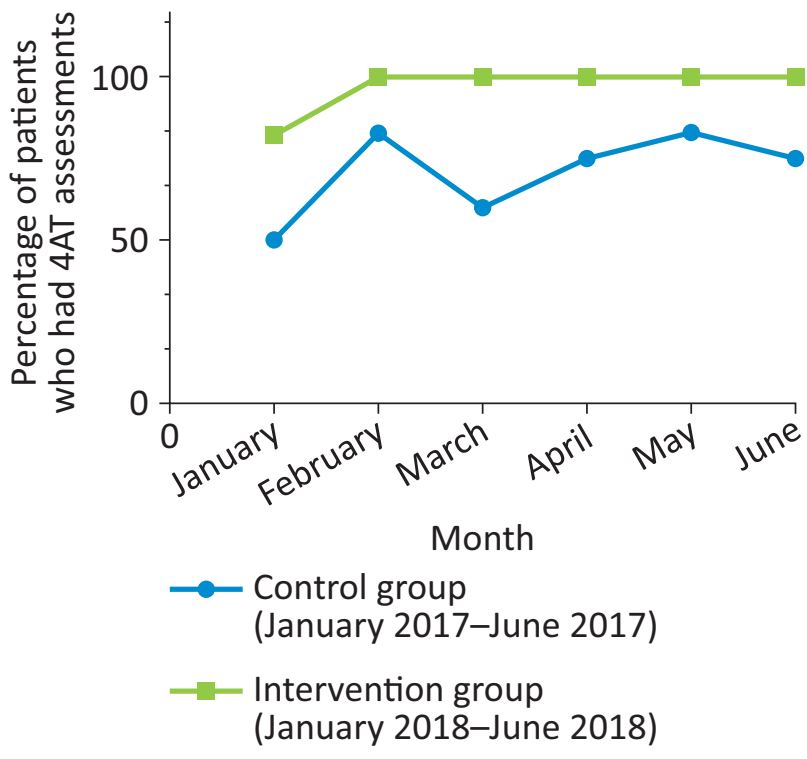

Fig 3. Percentage of 4AT assessments completed during hospital admission in control and intervention groups.

\section{Ethical considerations}

This project was registered with the trust as a quality improvement project and approved locally. All guidelines were developed in accordance with trust policy and approved for use.

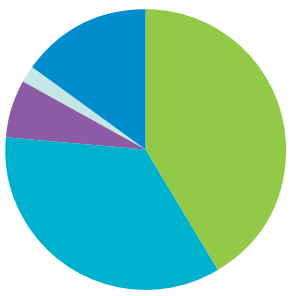

$41.67 \%$ doctor

$35.00 \%$ physiotherapist

$6.67 \%$ nurse

\section{$1.67 \%$ occupational therapist}

$15.00 \%$ other

Fig 4. Breakdown of percentages of 4AT completed by different members of the multidisciplinary team.

\section{Results}

Forty-five patients were admitted to the surgical wards ( $n=2)$ with a NOF fracture in January-June 2018 (post-intervention group). Forty were admitted in the period January-June 2017. All were included in the study. Both groups were made of similar patient populations (see Table 1), and had a higher proportion of women than men, in keeping with higher prevalence of NOF fracture in women.

There was a significant increase in the use of the 4AT; completed at least once during admission in $97 \%$ of patients in the postintervention group compared to $71 \%$ in the control group (95\% confidence interval 16.66-35.34; $p=0.0008$; see Fig 3). These were completed by a variety of healthcare professionals (see Fig 4).

There was no difference in the rate of delirium reported in each group $(47.5 \%$ in the control group and $44 \%$ in the postintervention group). Of patients diagnosed with delirium in the control group, $26 \%$ were diagnosed without the use of the 4 AT. Of delirium cases in the post-intervention group, $100 \%$ were

\begin{tabular}{|c|c|c|c|c|}
\hline & $\begin{array}{l}\text { Control group } \\
\text { (January-June 2017) }\end{array}$ & $\begin{array}{l}\text { Intervention group } \\
\text { (January-June 2018) }\end{array}$ & Significance & $\begin{array}{l}\text { Statistical } \\
\text { test }\end{array}$ \\
\hline Length of stay, days, mean & 25.09 & 24.3 & $\begin{array}{l}\text { Not significant } p=0.8704 \\
(95 \% \text { CI }-10.32-8.753)\end{array}$ & t-test \\
\hline $\begin{array}{l}\text { Mortality, patients not surviving } \\
\text { admission, \% }\end{array}$ & 12 & 8.89 & $\begin{array}{l}\text { Not significant } \\
(p=0.5891)\end{array}$ & $\chi^{2}$ \\
\hline $\begin{array}{l}\text { Patients being discharged to a different } \\
\text { destination from admission, } \%\end{array}$ & 12.5 & 17.78 & $\begin{array}{l}\text { Not significant } \\
(p=0.4988)\end{array}$ & $\chi^{2}$ \\
\hline
\end{tabular}

$\mathrm{CI}=$ confidence interval. 
a

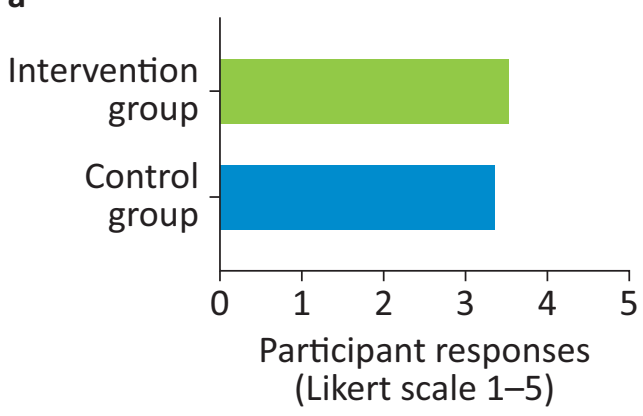

b

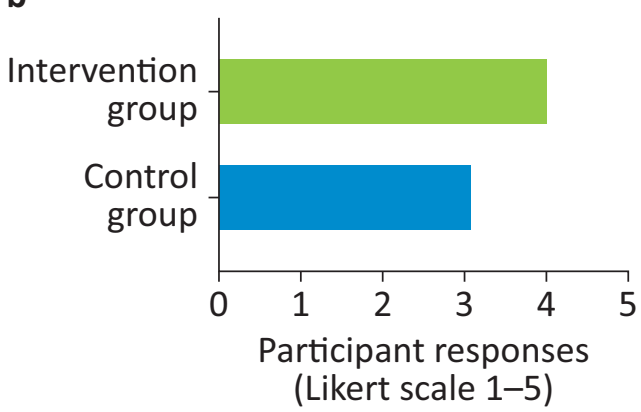

C

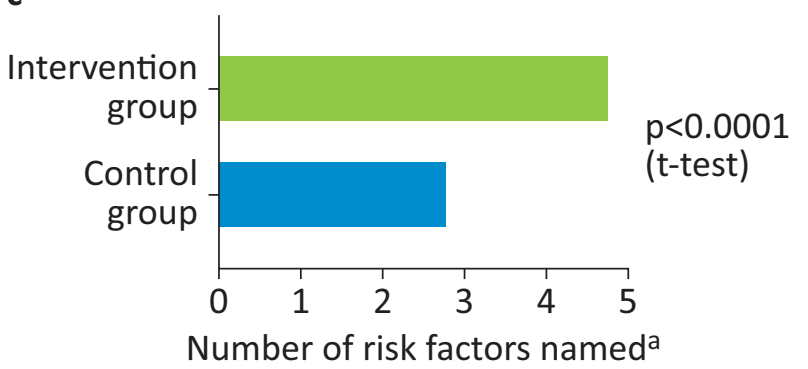

d

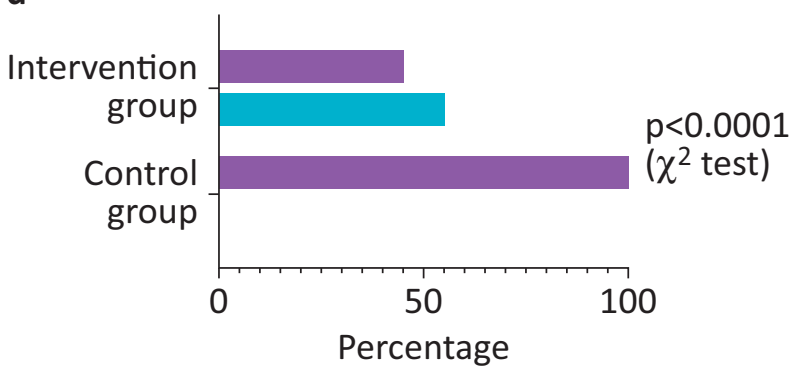

Able to name guidelines Not able to name guidelines

Fig 5. Results from staff survey data. a) Confidence in making delirium diagnosis. b) Confidence in explaining delirium. c) Number of delirium risk factors named by participants. d) Number of doctors able to name national or local guidelines. ${ }^{a}=$ as defined in the National Institute for Health and Care Excellence guidelines.

diagnosed using the 4AT. In those who were diagnosed with delirium in the control group, $42 \%$ had 'delirium' coded on the discharge summary. In those diagnosed with delirium in the postintervention group $55 \%$ were coded for on the discharge summary $(p=0.6356)$. There were no statistically significant difference in the secondary outcome measures (see Table 2).
Staff survey data was also analysed (see Fig 5). Questionnaires from the control group $(n=45)$ were compared to questionnaires from the post-intervention group ( $n=15)$. The control group contained responses from doctors, occupational therapists, physiotherapists, rehabilitation assistants and nursing staff. The post-intervention group contained responses from doctors, occupational therapists, physiotherapists and rehabilitation assistants. These data showed that there was a significant increase in staff confidence in explaining delirium and ability to name delirium risk factors. Doctors' ability to name specific regional or national guidelines also increased.

\section{Discussion}

The data indicate that the development of the three-stage pathway and associated implementation strategy increased staff knowledge of delirium and use of the 4AT assessment tool. The value of the $4 A T$ in the detection of postoperative delirium over other tools was recognised in 2016 National Hip Fracture Database annual report and subsequent inclusion in the NHS England and NHS Improvement neck of femur best practice tariff in 2017. ${ }^{10,11}$ These data also indicate that the project successfully engaged the multidisciplinary team (MDT). The importance of multicomponent, non-pharmacological interventions in the management of delirium is well established. ${ }^{12}$ By ensuring the engagement of the MDT within this educational programme, it may facilitate subsequent improvement in the management and delivery of such non-pharmacological interventions.

The rate of delirium recorded in both groups were in keeping with published rates of delirium ( $47.5 \%$ in the control group and $44 \%$ in the post-intervention group). ${ }^{1}$ The similarity in rates between the two groups may seem surprising, however, in the control group only $74 \%$ of these cases were diagnosed with the use of the $4 \mathrm{AT}$, compared to $100 \%$ in the post-intervention group. This may indicate improved diagnostic accuracy in the post-intervention group. This is particularly important when patients are not being assessed by specialist teams (such as in out-of-hours settings) as $4 A T$ is valid after only limited training. The use of the $4 A T$ may therefore allow more accurate earlier diagnosis. Given the complexity and cost associated with managing patients with delirium, the 4AT may also help target resources more appropriately.

The authors recognise the limitations of the study. The small number of patients in each group and in the questionnaire responses mean some changes and trends may go unrecognised. Further work looking at a larger patient group would allow the pathway to be tailored to other clinical settings. Data from the staff surveys showed increased knowledge of managing delirium but no significant change in confidence in diagnosis. This may be due to the timing of the post-intervention survey; which was completed after the small group lectures but before the implementation of the publicity campaign. The campaign was designed specifically to boost confidence and increase staff knowledge. The authors were also unable to assess the use of the PINCH ME mnemonic. This was because the PINCH ME pathway was enacted by multiple members of the MDT throughout a patient's admission and there was no tool to record this in the electronic patient record. This was a major limitation of the study and became the focus of subsequent PDSA cycles.

The project remains active. Following the initial work, the focus of the steering group has moved to management and subsequent outcome measures. New initiatives have included embedding of 
the PINCH ME mnemonic into the electronic computer system development of delirium guidelines and a quick reference guide for the trust intranet, regular teaching sessions for rotating members of the MDT, and regular publicity campaigns (often centred around national events such as National Delirium Day). It is hoped that this will ensure sustainability and improve key performance indicators. The analysis of these data is ongoing

This work shows that development of an effective clinical pathway and simple educational programme can increase staff understanding of delirium, and increase the use of the 4AT screening tool. Further work is needed to demonstrate if it is successful in improving the management of these patients and key performance indicators in this high-risk group.

\section{References}

1 Rudolph JL, Marcantonio ER. Postoperative delirium: acute change with long-term implications. Anesth Analg 2011;112:1202-11.

2 Marcantonio ER, Flacker JM, Michaels M, Resnick NM. Delirium is independently associated with poor functional recovery after hip fracture. J Am Geriatr Soc 2000;48:618-24.

3 Dewar B, Bond P, Miller M, Gouldie K. THINK Delirium. London: The Kings Fund, 2013.

4 Young J, Murthy L, Westby M, Akunne A, O'Mahony R. Diagnosis, prevention, and management of delirium: summary of NICE guidance. BMJ 2010;341:c3704
5 Bellelli G, Morandi A, Davis DHJ et al. Validation of the 4AT, a new instrument for rapid delirium screening: a study in 234 hospitalised older people. Age and Ageing 2014;43:496-502.

6 Sands MB, Dantoc BP, Hartshorn A, Ryan CJ, Lujic S. Single Question in Delirium (SQiD): testing its efficacy against psychiatrist interview, the Confusion Assessment Method and the Memorial Delirium Assessment Scale. Palliat Med 2010;24:561-5.

7 National Institute for Health and Care Excellence. Delirium: prevention, diagnosis and management. Clinical guideline [CG103]. NICE, 2019.

8 Dixon M. Assessment and management of older patients with delirium in acute settings. Nurs Older People 2018;30:35-42.

9 Taylor MJ, McNicholas C, Nicolay C et al. Systematic review of the application of the plan-do-study-act method to improve quality in healthcare. BMJ Qual Saf 2014;23:290-8.

10 Boulton C, Bunning T Burgon V et al. National Hip Fracture Database (NHFD) annual report 2016. London: Royal College of Physicians, 2016.

11 Boulton C, Bunning T, Johansen A et al. National Hip Fracture Database (NHFD) annual report 2017. London: Royal College of Physicians, 2017.

12 Martinez F, Tobar C, Hill N. Preventing delirium: should nonpharmacological, multicomponent interventions be used? A systematic review and meta-analysis of the literature. Age and Ageing 2015;44:196-204.

Address for correspondence: Dr Letitia Dormandy, Homerton University Hospital NHS Foundation Trust, Care of the Elderly, Homerton Row, London E9 6SR, UK.

Email: I.dormandy@nhs.net

\section{Royal College of Physicians}

\section{RCP journals now available in PubMed Central}

\author{
Both ClinMed and Future Healthcare Journal are now available \\ in PubMed Central, a web-based, free full-text archive of journal \\ literature for all biomedical and life sciences. \\ Inclusion of the content back to the very first issue of both \\ journals provides readers with greater access to valuable \\ scholarly content and makes finding articles easier. It also means \\ that journal content is now part of a permanent and freely \\ accessible archive, managed by the National Library of Medicine.
}

FHJ can be accessed at: www.ncbi.nlm.nih.gov/pmc/journals/3619 Clin Med at: www.ncbi.nlm.nih.gov/pmc/journals/2945
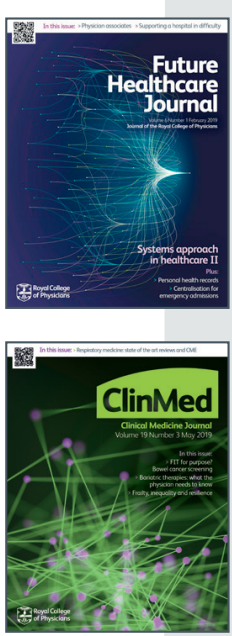